Article

\title{
Single-Switch LED Post-Regulator Based on a Modified Class-E Resonant Converter with Voltage Clamp
}

\author{
Javier Ribas*(D), Pablo J. Quintana, Jesus Cardesin, Antonio J. Calleja ${ }^{\mathbb{D}}$ and \\ Emilio Lopez-Corominas \\ CE3I2 Group, Department of Electrical Engineering, University of Oviedo, 33204 Gijon, Spain \\ * Correspondence: ribas@uniovi.es; Tel.: +34-985-182-550
}

Received: 25 June 2019; Accepted: 15 July 2019; Published: 16 July 2019

check for updates

\begin{abstract}
The strict restrictions imposed both by mandatory regulations and by the recommendations contained in current standards have led to the fact that most commercially available LED ballasts nowadays use two-stage topologies. The first stage is intended to comply with the harmonics standards and the second stage is used to control the LED current and reduce the low frequency ripple. In this work, a new DC-DC resonant converter topology is presented. This topology is derived from a modified Class-E resonant inverter by adding a clamping diode. This diode achieves a double goal: it limits the maximum switch voltage and works as a power recirculating path. This way, the proposed topology behaves as a loss-less impedance placed in series with the LED thus allowing to control the output power. This converter maintains the extremely small switching losses inherent to the Class-E inverter while reducing the voltage stress across the switch. This work presents a simplified design methodology based on the fundamental approach. This methodology was used to design and build a DC-DC post-regulator for a $40 \mathrm{~W}$ LED lamp. The results obtained with the laboratory prototype show that this circuit can be used to stabilize and dim the LED current while maintaining very small losses. The measured efficiency was $95.7 \%$ at nominal power and above $90 \%$ when dimmed down to $25 \%$.
\end{abstract}

Keywords: LED driver; high efficiency LEDs; solid state lighting; resonant DC-DC converter; Class-E inverter; single-switch topology; voltage clamp

\section{Introduction}

Since the appearance of the first high-efficiency blue LEDs in 1993, solid-state lighting devices have experienced a strong advance. In the last few years, almost every technical feature of LEDs has been greatly improved. For example, the evolution of the semi-conductor technology used to produce blue light, together with the improvement of the characteristics of the phosphors used to turn that blue light into the desired white light, allow obtaining a color spectrum suited even for applications where exceptional color reproduction is required (e.g., illumination of paintings). On the other hand, the optics used nowadays in LED lamps allow obtaining wide light beams with a good color consistency across the entire illuminated area [1]. The thermal behavior and the light depreciation along the LED life have also been improved thus making possible the construction of high power modules [2,3]. For example, there can be found currently in the market chip-on-board (COB) LED modules of more than $200 \mathrm{~W}$. Another important feature of LEDs is their fast response and ease of control, enabling the implementation of complex control strategies [4-6]. All these improvements, combined with the continuous cost reduction that has taken place in recent years, have made LEDs the preferred choice for most lighting applications nowadays. 
The availability of better light sources has also been considered by many public administrations to push the specifications required for new installations to higher levels $[7,8]$. Furthermore, even the metrics used to characterize light sources have had to adapt to the new possibilities associated with the use of solid-state lighting systems. For example, the color rendering index (CRI) is considered insufficient to characterize the color reproduction of a light source based on LEDs. Many manufacturers are using the color metrics described in IES TM-30-18 in their product datasheets [9]. In the same way, new light flicker standards have also appeared setting stricter limits that could hardly be met using traditional lighting systems (e.g., IEEE 1789 [10]).

The need to comply with both the input harmonic and the output flicker standards have led manufacturers to lean towards two-stage topologies. The first stage is used for power factor correction (PFC) and is needed to comply with the standard IEC 61000-3-2. The second stage is used to control the LED current and minimize the light flicker.

The most commonly used topologies for PFC in solid-state lighting systems are the flyback and boost converters. However, in the bibliography there can be found examples where almost every topology suited for PFC are used as well [11,12]. The second stage can be used for several purposes: flicker reduction, current balancing across multiple LED strings, adding dimming capability, or implementing light modulation techniques for visible light communications [13]. Different solutions can be found in the literature concerning these mentioned features. For instance, current equalization in multi-string LED lamps can be performed by optimized dissipative solutions [14] as well as by specific power topologies that maximize the efficiency [15]. When the main goal is to control the LED current in order to either provide dimming or to eliminate the low frequency flicker, there are solutions based in resonant [16] and non-resonant configurations [17,18]. In some designs, the bulk capacitor required in the first stage for PFC is made using plastic film instead of electrolytic capacitors. In this case, the second stage has to handle a greater input voltage ripple in order to reduce the size of the required capacitor. This change allows increasing the driver lifetime compared to using a low-cost electrolytic capacitor [19,20].

Present work describes a new resonant topology especially suited for this purpose. The proposed circuit is based on the series regulator concept described in Figure 1. The current through the LED is controlled by a filter inductance $L_{F}$ followed by a high frequency converter that behaves as a variable loss-less resistor. The power handled by this converter is sent back to the input by means of the recycling diode $D_{R}$. The amount of power recycled will be directly related to the converter voltage ratio. If the input and output voltages are equal the power recycled will be null.

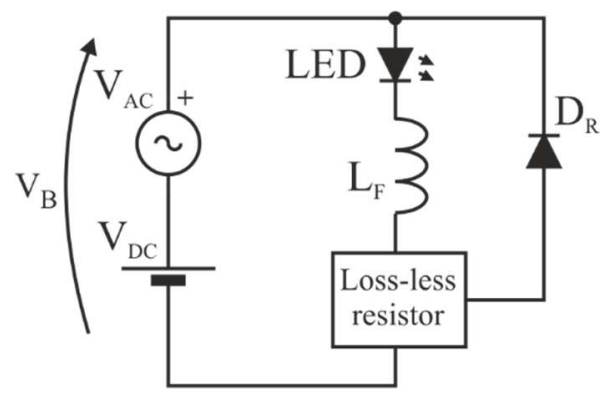

Figure 1. Operation of the series current regulator.

The simplified schematic of the proposed topology is shown in Figure 2. This topology can be derived from two different basic topologies: the buck DC-DC converter or the class-E inverter. One of the techniques used in the bibliography to derive resonant converters from standard DC-DC configurations is based on adding resonant networks. These networks are used to reduce switching losses thus enabling the possibility to operate at higher frequencies without compromising the efficiency [21,22]. The proposed topology can be derived from a buck converter by adding a soft-switching resonant 
network consisting of two branches in parallel: one formed only by capacitor $C_{P}$, and another by the inductance $L_{R}$ in series with capacitor $C_{R}$ (See Figure 2).

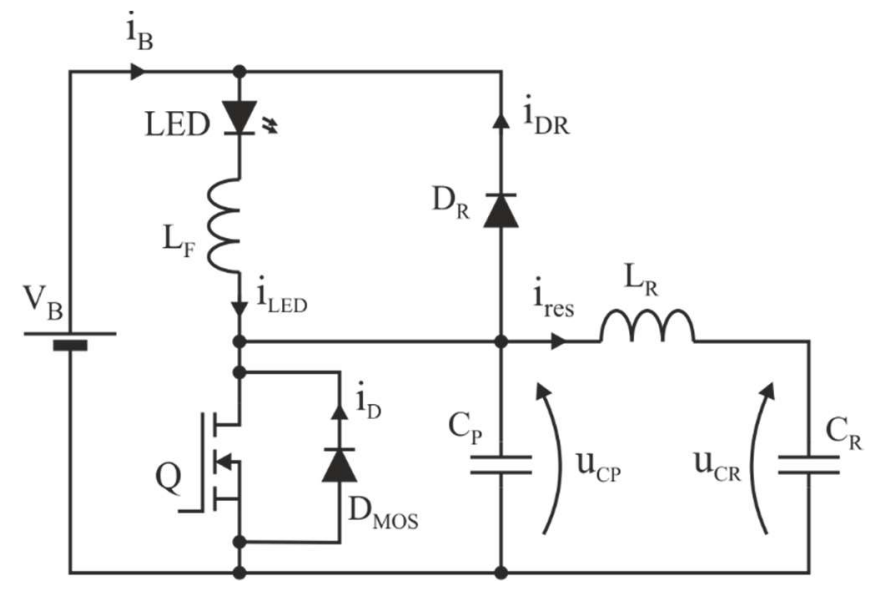

Figure 2. Schematics of the proposed topology.

In this circuit, $\mathrm{L}_{\mathrm{R}}$ and $\mathrm{C}_{\mathrm{R}}$ constitute the main resonant network, handling an almost sinusoidal current $i_{\text {res. }}$. This current allows a smooth charge and discharge of capacitor $C_{P}$ between zero and the bus voltage $V_{B}$. The key aspect for proper operation of this circuit is to ensure a complete discharge of capacitor $C_{P}$ before the switch $Q$ is turned on. If this zero-voltage-switching (ZVS) condition is not met, the efficiency will drop significantly as the energy stored in $C_{P}$ at turn-on will be dissipated in the mosfet, causing strong current spikes. On the other hand, capacitor $C_{P}$ ensures a small $\mathrm{dv} / \mathrm{dt}$ across transistor $\mathrm{Q}$ during the turn-off transient thus reducing the switch-off losses. This behavior is also found in the standard Class-E inverter configuration, allowing these circuits to be used at very high frequencies [23].

One of the main disadvantages of the Class-E inverter and some similar topologies [16], is the high peak-to-average ratio of the mosfet voltage. In many designs, this ratio can be higher than five making necessary the use of high-voltage transistors. In the proposed topology, the maximum voltage across the mosfet is equal to the bus voltage $V_{B}$ due to the addition of the clamping diode $D_{R}$. Similar alternatives where explored in the bibliography for other circuit configurations not intended for LED supply [24].

The most similar topology described in prior literature is the modified Class-E converter found in [16]. In this circuit, the AC output of a standard Class-E inverter is rectified and sent back to the input obtaining a DC-DC series regulator. However, no voltage clamping is used and the mosfet voltage can be considerably higher.

The remainder of the paper will be organized as follows. In first place, a detailed description of the operation of the proposed circuit will be made. This description will be followed by a simplified analysis based on the fundamental approach. The resonant current $i_{\text {RES }}$ will be approximated as a pure sinusoidal signal to obtain a set of design charts. In section four, basic hints to design a circuit based in these charts will be discussed. Next, a sensitivity analysis will be carried out. This analysis is intended to provide a general idea on how the control must work to compensate for small-signal perturbations at the input or the load. Finally, the laboratory prototype will be described together with the experimental results obtained at different operating conditions. The main conclusions of this work will be given at the last section.

\section{Circuit Operation}

To simplify the circuit analysis, the resonant current will be approximated as a pure sinusoidal signal and the LED current ripple will be neglected. Figure 3 shows the main waveforms of the proposed circuit based in those assumptions. The most important trace to understand how the 
circuit operates is the $\mathrm{i}_{\mathrm{LED}} \mathrm{i}_{\text {res }}$ shown in second place. When the transistor is switched-off (indicated as $t_{\mathrm{sw} \text {-off }}$ in the figure), capacitor $C_{P}$ starts charging until it reaches the bus voltage $V_{B}$. Once this happens, the clamping diode $\mathrm{D}_{\mathrm{R}}$ starts conducting. During this interval, the current handled by this diode is equal to $i_{L E D}-i_{\text {res, }}$, and the current through the source $i_{B}$ is equal to the resonant current $i_{\text {res }}$, thus providing a steep change in the input current at the instant when the clamping diode starts conducting. When $\mathrm{i}_{\mathrm{LED}} \mathrm{i}_{\text {res }}$ goes down to zero, the clamping diode turns off. At this point, voltage $\mathrm{u}_{\mathrm{CP}}$ starts decreasing due to its negative current balance (negative shaded area). Once $\mathrm{C}_{\mathrm{P}}$ is completely discharged, the current starts flowing through the mosfet body diode. The transistor should be turned on before this condition is lost in order to achieve ZVS. When the clamping diode is in the off state, the current handled by the input source is equal to the LED current $i_{\text {LED }}$.

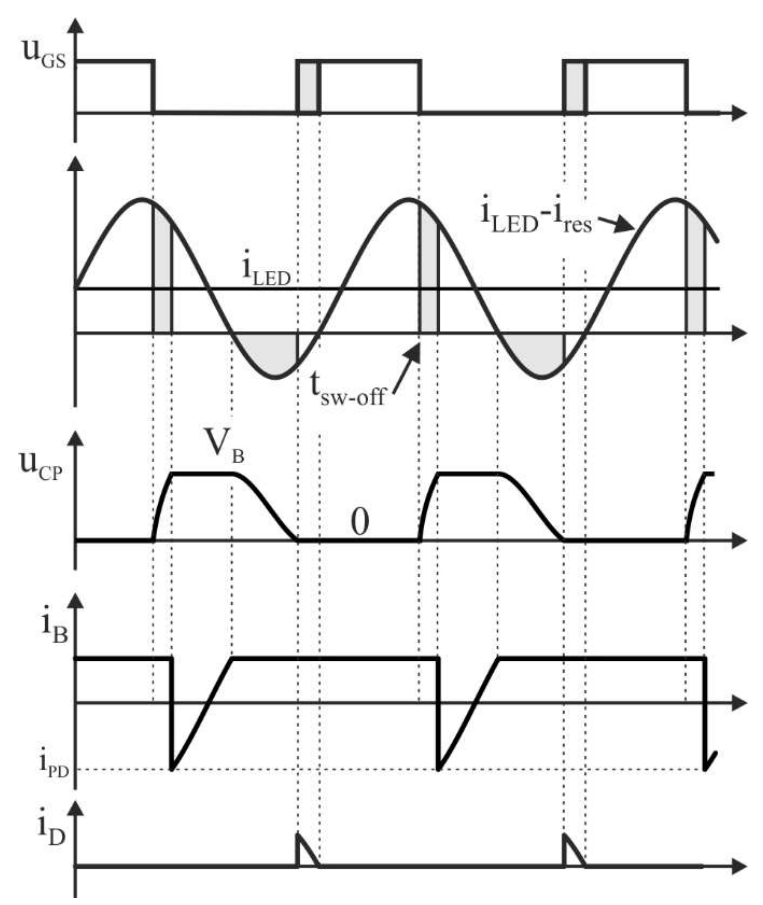

Figure 3. Basic waveforms of the proposed series regulator.

\section{Circuit Analysis Based on the Fundamental Approach}

To obtain the simplified equations and design charts that describe the circuit operation the next four steps will be followed:

- Identify the angles where changes in the operating mode take place

- $\quad$ Obtain the normalized expression of the mosfet voltage $\mathrm{u}_{\mathrm{CP}}$

- Determine the relations between angles and normalized circuit parameters

- Numerically solve the set of non-linear equations and depict the normalized design charts.

The detailed representation of the main circuit waveforms during one switching cycle is shown in Figure 4. 


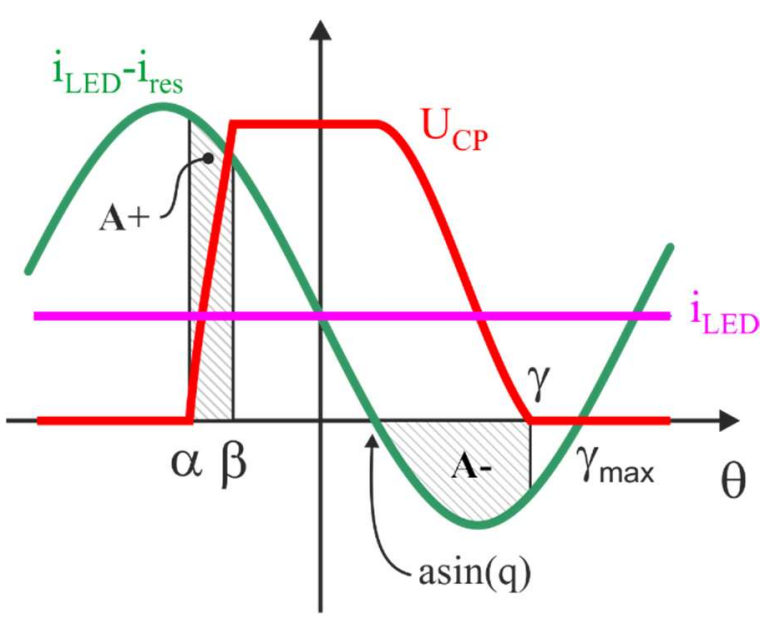

Figure 4. Detail of the operation of the proposed circuit.

In this figure, the angle reference is set at the zero crossing of the resonant current. $\alpha$ angle defines the point where the transistor switches off. $\beta$ is the angle where $C_{P}$ reaches the bus voltage and the clamping diode $D_{R}$ turns on. When the current $i_{L E D} i_{\text {res }}$ becomes negative (at $\operatorname{asin}(q)$ ), capacitor $C_{P}$ starts discharging until it reaches zero at $\gamma$ angle. The positive and negative shaded areas marked as $\mathrm{A}+$ and $\mathrm{A}-$ in Figure 4 must be equal at steady state, as the average capacitor current is null. This condition cannot be fulfilled if the angle $\gamma$ is greater than the $\gamma_{\max }$ angle indicated in the figure.

This behavior can be described by the following formula:

$$
U_{C P}(t)=\left\{\begin{array}{ll}
\frac{1}{C_{P}} \int_{\alpha / \omega}^{t}\left(i_{L E D}-I_{\text {res }(\text { peak })} \cdot \sin \left(\omega \cdot t_{a}\right)\right) \cdot d t_{a} & \text { if } \frac{\alpha}{\omega}<t \leq \frac{\beta}{\omega} \\
V_{B} & \text { if } \frac{\beta}{\omega}<t \leq \frac{a \sin (q)}{\omega} \\
V_{B}+\frac{1}{C_{P}} \int_{a \sin (q) / \omega}^{t}\left(i_{L E D}-I_{\text {res }(\text { peak })} \cdot \sin \left(\omega \cdot t_{b}\right)\right) \cdot d t_{b} & \text { if } \frac{a \sin (q)}{\omega}<t \leq \frac{\gamma}{\omega} \\
0 & \text { otherwise }
\end{array},\right.
$$

where $\omega$ corresponds with the operating angular frequency and $I_{\text {res (peak) }}$ is the peak value of the resonant current. To normalize the previous expression, parameter $q$ will be defined as:

$$
q=\frac{i_{L E D}}{I_{\text {res }(\text { peak })}}
$$

Expression (1) can then be transformed into:

$$
M_{C P}(\theta)=\left\{\begin{array}{ll}
\int_{\alpha}^{\theta}\left(1-q^{-1} \sin \left(\theta_{a}\right)\right) \cdot d \theta_{a} & \text { if } \alpha<\theta \leq \beta \\
M_{B} & \text { if } \beta<\theta \leq a \sin (q) \\
M_{B}+\int_{a \sin (q)}^{\theta}\left(1-q^{-1} \sin \left(\theta_{b}\right)\right) \cdot d \theta_{b} & \text { if } \operatorname{asin}(q)<\theta \leq \gamma \\
0 & \text { otherwise }
\end{array},\right.
$$

by means of the following definition:

$$
U_{C P}(t)=\frac{I_{r e s(p e a k)}}{C_{P} \cdot \omega} M_{C P}(\omega \cdot t) .
$$

The normalized expression of the bus voltage $M_{B}$ can be calculated using:

$$
M_{B}=\int_{\alpha}^{\beta}\left(1-q^{-1} \sin \left(\theta_{a}\right)\right) \cdot d \theta_{a} .
$$


There are several restrictions that apply to Expression (3). These restrictions can be used to obtain a set of three equations that define the basic relations between circuit parameters. These equations will be used to obtain the main design charts.

In first place, the voltage balance across $C_{P}$ capacitor means that areas $\mathrm{A}+$ and $\mathrm{A}-$ must be equal, (see Figure 4). This condition provides the following equation:

$$
F 1(q, \alpha, \beta, \gamma)=\beta-\alpha+\frac{1}{q}(\cos (\beta)-\cos (\alpha))+\gamma-\operatorname{asin}(q)+\frac{\cos (\gamma)-\sqrt{1-q^{2}}}{q}=0 .
$$

The second equation will be obtained by means of the input-to-output power balance. Considering that the input and output powers must be equal, the following formula is obtained:

$$
F 2(q, \beta, \kappa)=\frac{\kappa}{2 \pi}\left(2 \pi-a \sin (q)+\beta+\int_{\beta}^{a \sin (q)} \frac{1}{q} \sin (\theta) \cdot d \theta\right)-1=0,
$$

where parameter $k$ is defined as:

$$
\kappa=\frac{V_{B}}{V_{L E D}} .
$$

The third equation will be obtained using the restriction that comes from the fact that the $L_{R}-C_{R}$ resonant tank cannot handle active power. Therefore, the $M_{C P}$ component of the fundamental harmonic that is in phase with the resonant current must be zero:

$$
F 3(q, \alpha, \beta, \gamma)=\int_{-\pi}^{\pi} M_{C P}(\theta, q, \alpha, \beta, \gamma) \cdot \sin (\theta) \cdot d \theta=0 .
$$

Expressions (6), (7), and (9) are defined as functions of 5 parameters, that is: $q, \alpha, \beta, \gamma$, and $k$.

$$
\begin{gathered}
F 1(q, \alpha, \beta, \gamma)=0 \\
F 2(q, \beta, \kappa)=0 \\
F 3(q, \alpha, \beta, \gamma)=0
\end{gathered}
$$

These three equations allow to determine the angles that define the circuit operation $(\alpha, \beta$, and $\gamma)$ as functions of parameters $q$ and $k$. The charts shown in Figure 5 were obtained by numerically solving Equations (10)-(12) for different values of $q$ and $k$. According to theses charts, there are several conclusions that can be extracted. As it was stated before, transistor switch-on must take place between the instants defined by $\gamma$ and $\gamma_{\max }$ to achieve ZVS. For $k=2$, as both angle values are coincident, there is no control margin and the transistor must be turned on at $\gamma$.

Therefore, $k=2$ defines the theoretical maximum ratio between the input and output voltages that can be used. For higher $k$ values it is not possible to fully discharge capacitor $C_{P}$ and maintain ZVS, giving rise to high switching losses. On the other hand, having a significant difference between $\gamma$ and $\gamma_{\max }$ means that the reactive power handled by the converter is high, increasing the losses in all circuit components. Besides, for small $k$ values, the $q$ range that can be used is limited to the area where $\alpha$ and $\beta$ are different thus the shaded area shown in Figure 5a should be avoided.

In fact, one of the most important limitations of the proposed circuit is that the input-to-output ratio must be between 1.2 and 2 for proper operation. 


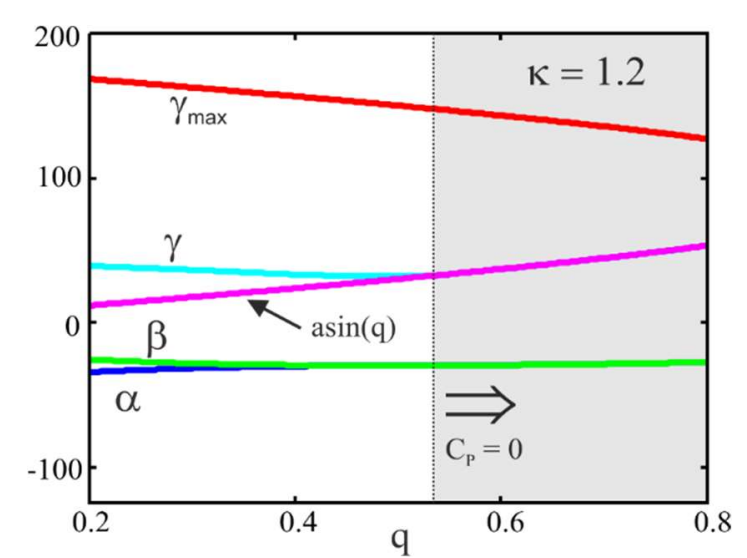

(a)

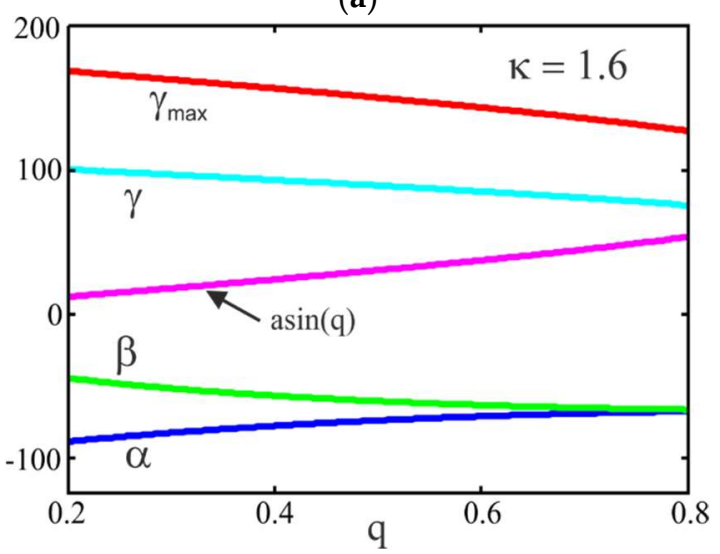

(c)

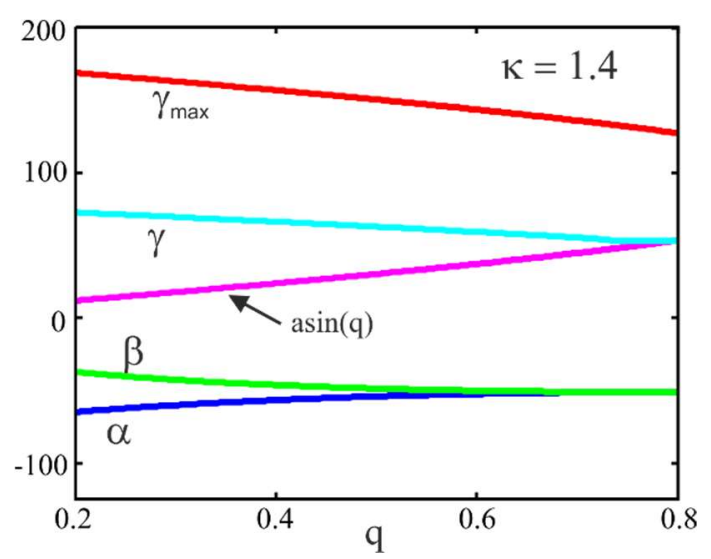

(b)

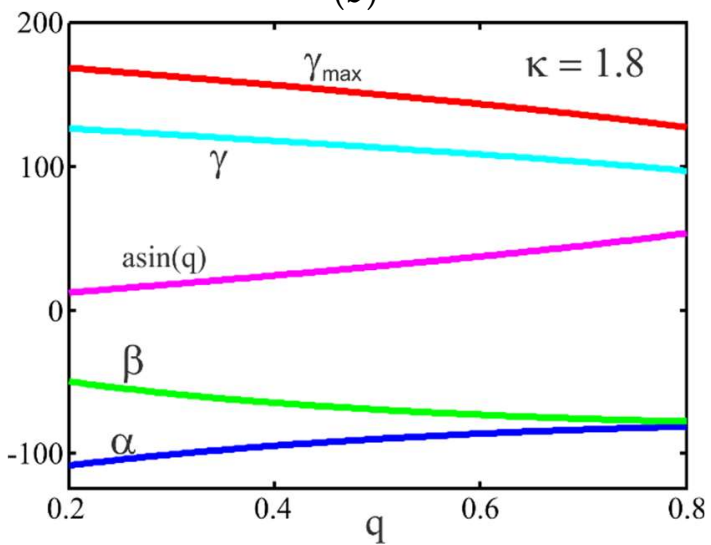

(d)

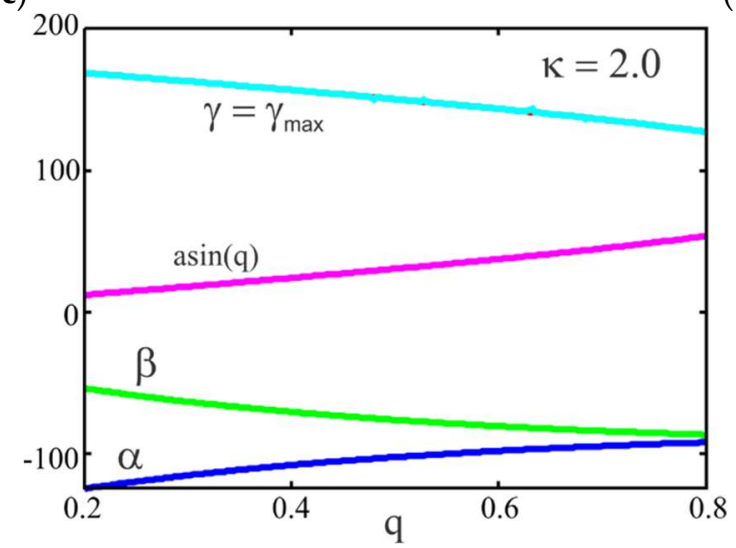

(e)

Figure 5. Variation of the angles $\alpha, \beta, \operatorname{asin}(q), \gamma \mathrm{y} \gamma_{\max }$ as a function of $q$ for different $k$ values: (a) $k$ equal to 1.2 ; (b) $k$ equal to 1.4 ; (c) $k$ equal to 1.6 ; (d) $k$ equal to 1.8 ; and (e) $k$ equal to 2.0 .

\section{Proposed Design Methodology}

The best way to illustrate how the design can be made is by means of a practical example. For that purpose, a $40 \mathrm{~W}$ lamp with a rated voltage of $80 \mathrm{~V}$ will be used. The lamp specifications and the basic circuit parameters of this example are summarized on Table 1 . The circuit will be used to reduce the low frequency ripple in the LED lamp and provide dimming capability. As it was addressed in previous section, parameter $k$ must be between 1.2 and 2 for proper operation. In this example, the maximum $k$ will be set at 1.9 and the minimum at 1.3, with a nominal value of 1.6. This allows handling a $16 \mathrm{~V}$ low frequency ripple with a nominal bus voltage of $128 \mathrm{~V}$. 
Table 1. Design example specifications.

\begin{tabular}{|c|c|c|c|}
\hline \multicolumn{4}{|c|}{ Basic Circuit Specifications } \\
\hline Nominal input voltage & \multicolumn{2}{|c|}{ Input voltage excursion } & Nominal frequency \\
\hline $128 \mathrm{~V}$ & \multicolumn{2}{|c|}{ $\pm 16 \mathrm{~V}$ (peak) } & $200 \mathrm{kHz}$ \\
\hline Nominal output current & \multicolumn{2}{|c|}{ Output voltage } & Nominal output power \\
\hline $0.5 \mathrm{~A}$ & \multicolumn{2}{|c|}{$80 \pm 4 \mathrm{~V}$} & $40 \mathrm{~W}$ \\
\hline \multicolumn{4}{|c|}{ LED Lamp Characteristics } \\
\hline Model & Manufacturer & Nominal current & Number of LEDs \\
\hline Oslon SSL80 & Osram & $0.8 \mathrm{~A}$ & 24 \\
\hline \multicolumn{4}{|c|}{ Selected Design Parameters } \\
\hline$q$ & \multicolumn{2}{|c|}{$\kappa$} & $v$ \\
\hline 0.4 & \multicolumn{2}{|c|}{1.6} & 1.5 \\
\hline \multicolumn{4}{|c|}{ Circuit Parameters } \\
\hline$C_{P}$ & \multicolumn{2}{|c|}{$C_{R}$} & MOS \\
\hline $3.7 \mathrm{n}$ & \multicolumn{2}{|c|}{$6.8 \mathrm{nF}$} & IRF640 \\
\hline$L_{R}$ & \multicolumn{2}{|c|}{$L_{F}$} & Diode \\
\hline $141 \mu \mathrm{H}$ ETD29 3F3 & \multicolumn{2}{|c|}{2 mH ETD29 3F3 } & MUR120 \\
\hline
\end{tabular}

In this example, based on our experience, the nominal $q$ value was set to 0.4 , and the nominal operating frequency selected was $200 \mathrm{kHz}$. The nominal values of angles $\alpha, \beta$ and $\gamma$ can be obtained directly from Figure 5.

In the next paragraphs, the procedure to calculate the main circuit parameters will be addressed. In first place, integrating Equation (1) between $\alpha$ and $\beta$ the following relation is obtained:

$$
R \cdot \omega \cdot C_{P}=\frac{1}{\kappa} \cdot\left(\beta-\alpha+q^{-1}(\cos (\beta)-\cos (\alpha))\right),
$$

where $R$ value is defined as:

$$
R=\frac{V_{L E D}}{I_{L E D}} .
$$

This expression gives the $C_{P}$ value shown in Table 1 . On the other hand, the peak value of the fundamental component of voltage $U_{C P}$ must be equal to the equivalent impedance of the $L_{R}-C_{R}$ resonant tank $\left(Z_{\text {res }}\right)$ multiplied by $I_{\text {res }(\text { peak })}$. That is:

$$
\frac{V_{B}}{M_{B}} \cdot \frac{1}{\pi} \int_{-\pi}^{\pi} M_{C P}(\theta, q, \alpha, \beta, \gamma) \cdot \cos (\theta) \cdot d \theta=Z_{r e s} \cdot I_{\text {res }(\text { peak })} .
$$

This relation can also be expressed as:

$$
\frac{\kappa \cdot q}{M_{B}} \cdot \frac{1}{\pi} \int_{-\pi}^{\pi} M_{C P}(\theta, q, \alpha, \beta, \gamma) \cdot \cos (\theta) \cdot d \theta=\frac{Z_{r e s}}{R}
$$

This expression can be used to calculate $Z_{\text {res }}$ value at the nominal operating point. To determine the individual values of $L_{R}$ and $C_{R}$ an additional parameter must be defined:

$$
v=\omega^{2} \cdot L_{R} \cdot C_{R}
$$

Parameter $v$ corresponds to the ratio between the impedance at nominal frequency of the inductance $L_{R}$ referred to the impedance of $C_{R}$. 


$$
Z_{r e s}=\frac{1}{\omega \cdot C_{R}}(v-1)=\omega \cdot L_{R}\left(1-\frac{1}{v}\right)
$$

Normally, $Z_{\text {res }}$ impedance will have a dominant inductive behavior meaning that $v$ should be greater than one. However, if this parameter is much bigger than one, the $L_{R}-C_{R}$ will operate far from resonance and $i_{\text {res }}$ will be highly distorted. On the other hand, if $v$ is close to one, the circuit will be extremely sensitive to small changes in the operating frequency and tolerances in the component values. Besides, both $L_{R}$ and $C_{R}$ will handle higher voltages and present significantly higher losses. In this example, parameter $v$ was set to a trade-off value of 1.5. The calculated values of $L_{R}$ and $C_{R}$ are shown in Table 1.

Equation (15) can also be used to calculate the AC component of the voltage across the filter inductor $L_{F}$. The minimum $L_{F}$ value to obtain a ripple below $10 \%$ across the LED is $1.2 \mathrm{mH}$. In this design the selected $L_{F}$ was $2 \mathrm{mH}$.

The fundamental approach has been used in all previous steps of the proposed analysis. The validity of this approach can be confirmed by comparing the amplitude of higher order harmonics with the fundamental. An estimation of this distortion can be made by assuming that the voltage $U_{C P}$ can be approximated by Equation (1) even if there is a small distortion in the resonant current. Fourier analysis can then be used to estimate the higher order harmonics of $i_{\text {res }}$ and compare them to the fundamental. Figure 6 shows the ratio between the second harmonic and the fundamental as a percentage. As it can be observed, using the parameters of the previous example, the distortion of the second harmonic at nominal power is $7.3 \%$, thus the error derived of using the fundamental approach is quite small.

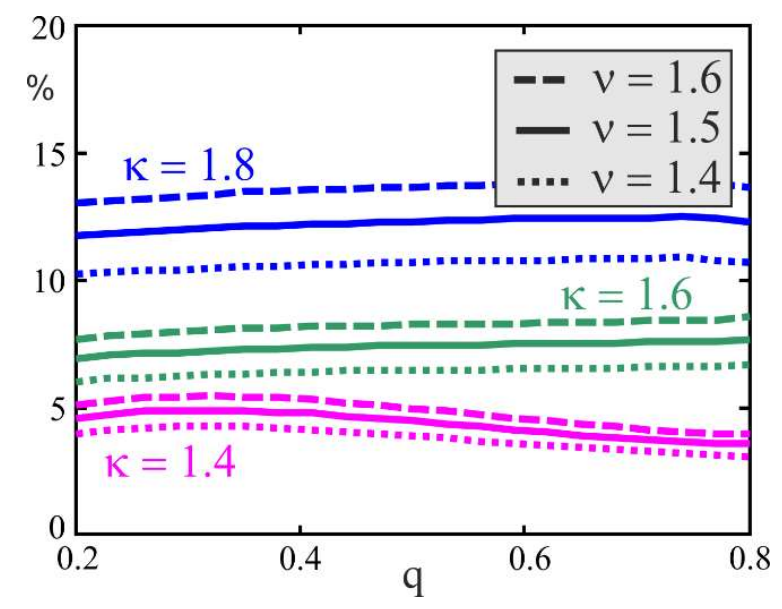

Figure 6. Ratio between the amplitudes of the second harmonic and the fundamental component of $i_{\text {res }}$.

\section{Small Signal Analysis of Proposed Converter}

As it was addressed before, the purpose of this circuit is to control the LED power with two objectives: reducing the low frequency ripple and providing dimming capability. Therefore, if either the bus or the lamp voltage change, the circuit must regulate the output power accordingly.

As in many resonant configurations, the only parameter that can be used to control the operating point of the proposed converter is the switching frequency. In this section, the non-linear equations obtained previously will be linearized in order to obtain the circuit sensitivity against changes in frequency, bus and lamp voltages.

To perform the small-signal analysis of the static characteristics, the Equations (10)-(12) will be combined with the Equations (13), (16), and (18). These last three equations were used to calculate $C_{P}$, $L_{R}$ and $C_{R}$, and can also be written as:

$$
F A(q, \alpha, \beta, \kappa)=R \cdot \omega \cdot C_{P}
$$




$$
\begin{gathered}
F B(q, \alpha, \beta, \gamma, \kappa, v)=R \cdot \omega \cdot C_{R} \\
F C(q, \alpha, \beta, \gamma, \kappa, v)=\frac{R}{\omega \cdot L_{R}}
\end{gathered}
$$

In the remaining of this paper, the caret sign $\left(^{\wedge}\right)$ will be used to identify small-signal variables, and sub-index ' 0 ' will be used to indicate steady-state values of all variables.

The main goal of this analysis is to obtain the relation between the small-signal values of the lamp power $\hat{p}$, lamp current $\hat{i}_{L E D}$, lamp voltage $\hat{v}_{L E D}$, bus voltage $\hat{v}_{B}$ and angular frequency $\hat{\omega}$. To do so, some of the parameters used in previous equations must be calculated as functions of these five small-signal variables. In first place, using the Definition (8), $\hat{\kappa}$ can be written as:

$$
\hat{\kappa}=\kappa_{0} \frac{\hat{v}_{B}}{V_{B 0}}-\kappa_{0} \frac{\hat{v}_{L E D}}{V_{L E D 0}}
$$

In a similar way, the lamp's equivalent resistance can be expressed as:

$$
\frac{\hat{R}}{R_{0}}=\frac{\hat{v}_{L E D}}{V_{L E D 0}}-\frac{\hat{i}_{L E D}}{I_{L E D 0}} .
$$

Linearizing (17), the small-signal value $\hat{v}$ is found to be proportional to $\hat{\omega}$ :

$$
\hat{v}=2 \cdot v_{0} \frac{\hat{\omega}}{\omega_{0}} .
$$

Lamp power $\hat{p}$ can be expressed as a linear function of $\hat{v}_{L E D}$ and $\hat{i}_{L E D}$ :

$$
\frac{\hat{p}}{P_{0}}=\frac{\hat{v}_{L E D}}{V_{L E D 0}}+\frac{\hat{i}_{L E D}}{I_{L E D 0}} .
$$

Combining previous expressions and definitions the following small-signal matrix equation is obtained:

$$
A \cdot\left[\begin{array}{c}
\hat{q} \\
\hat{\alpha} \\
\hat{\beta} \\
\hat{\gamma} \\
\hat{i}_{L E D}
\end{array}\right]=B \cdot\left[\begin{array}{c}
\hat{V}_{B} \\
\hat{V}_{L E D} \\
\hat{\omega}
\end{array}\right],
$$

where $A$ and $B$ matrixes are defined as:

$$
\begin{gathered}
A=\left[\begin{array}{ccccc}
\frac{\partial F 1}{\partial q} & \frac{\partial F 1}{\partial \alpha} & \frac{\partial F 1}{\partial \beta} & \frac{\partial F 1}{\partial \gamma} & 0 \\
\frac{\partial F 2}{\partial q} & 0 & \frac{\partial F 2}{\partial \beta} & 0 & 0 \\
\frac{\partial F}{\partial q} & \frac{\partial F 3}{\partial \alpha} & \frac{\partial F 3}{\partial \beta} & \frac{\partial F 3}{\partial \gamma} & 0 \\
\frac{\partial F A}{\partial q} & \frac{\partial F A}{\partial \alpha} & \frac{\partial F A}{\partial \beta} & 0 & F A_{0} \\
\frac{\partial F B}{\partial q} & \frac{\partial F B}{\partial \alpha} & \frac{\partial F B}{\partial \beta} & \frac{\partial F B}{\partial \gamma} & F B_{0}
\end{array}\right] \\
B=\left[\begin{array}{ccc}
0 & 0 & 0 \\
-\frac{\partial F 2}{\partial \kappa} \kappa_{0} & \frac{\partial F 2}{\partial \kappa} \kappa_{0} & 0 \\
0 & 0 & 0 \\
-\frac{\partial F A}{\partial \kappa} \kappa_{0} & \frac{\partial F A}{\partial \kappa} \kappa_{0}+F A_{0} & F A_{0} \\
-\frac{\partial F B}{\partial \kappa} \kappa_{0} & \frac{\partial F B}{\partial \kappa} \kappa_{0}+F B_{0} & -\frac{\partial F B}{\partial v} 2 v_{0}+F B_{0}
\end{array}\right] .
\end{gathered}
$$


Using Equation (26) and Definitions (22)-(25) it is possible to obtain each small-signal variable as a linear function of $\hat{V}_{B}, \hat{V}_{L E D}$, and $\hat{\omega}$. For example:

$$
\hat{i}_{L E D}=\left[\begin{array}{lllll}
0 & 0 & 0 & 0 & 1
\end{array}\right] \cdot A^{-1} \cdot B \cdot\left[\begin{array}{c}
\hat{V}_{B} \\
\hat{V}_{L E D} \\
\hat{\omega}
\end{array}\right] .
$$

This equation, combined with (25), can be used to obtain the lamp power sensitivity charts shown in Figure 7. These charts represent the ratio between the per-unit change in the lamp power $\hat{p}$, referred to the per-unit change in $\hat{V}_{L E D}, \hat{V}_{B}$, and $\hat{\omega}$, respectively.

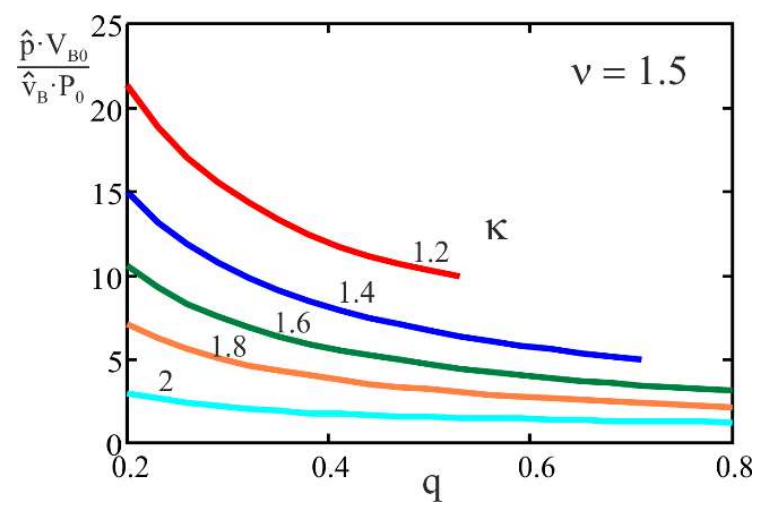

(a)

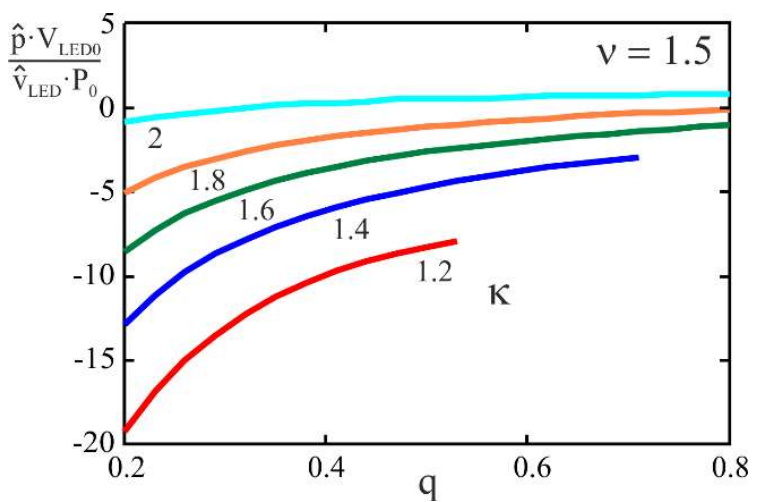

(b)

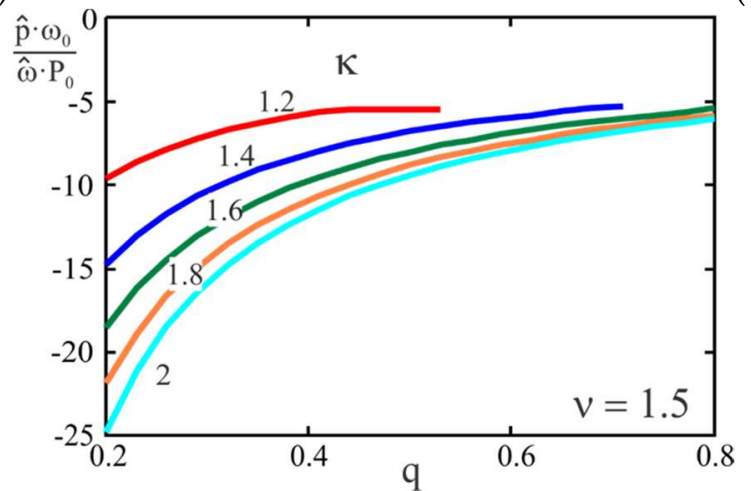

(c)

Figure 7. Small-signal output power static sensitivity as a function of $q$ for $v$ equal to 1.5: (a) Against changes in the input voltage; (b) Against changes in the LED voltage; (c) Against changes in the operating frequency.

One of the conclusions that can be extracted from these charts is that it is not possible to select an operating point where the power is independent of changes in the lamp voltage or the bus voltage. The best approach to this condition can only be obtained for $k$ close to two; but, as it was stated before, this should be avoided to maintain ZVS.

In the particular case of $q=0.4$ and $k=1.6$ used in the example described in previous section, a power against bus voltage sensitivity value of 5.6 can be obtained from Figure 7a. This value means that a variation of $1 \%$ in the bus voltage will lead to a change in the lamp power of $5.6 \%$. Therefore, the operating point is extremely sensitive to changes at the converter input thus the control circuit must sense and compensate for changes in the bus voltage. Figure $7 \mathrm{c}$ shows that the converter is highly sensitive to changes in the operating frequency. Therefore, the frequency excursion that will be required to control the operating point should be small. This is an interesting feature to minimize the size of the EMI filter that will be required. 
To optimize the control, the most precise solution would be sensing the LED power and adjusting the switching frequency in closed loop. The main problem for its practical implementation would be sensing the lamp current. An expensive isolated DC current sensor will be needed as the lamp is not referred to ground. To overcome this limitation, a simple feedforward control is proposed in this work.

The main problem of the feedforward control is that both the bus and the LED voltages have to be measured independently and two different gains must be used to control the frequency against variations in both parameters. In the proposed topology, sensing the difference between $V_{B}$ and $V_{L E D}$ is very easily done (see Figure 8 ). Therefore, if the sensitivities of the lamp power with respect to both parameters are the same, this single measurement would allow to adjust the frequency using a single voltage-to-frequency gain.

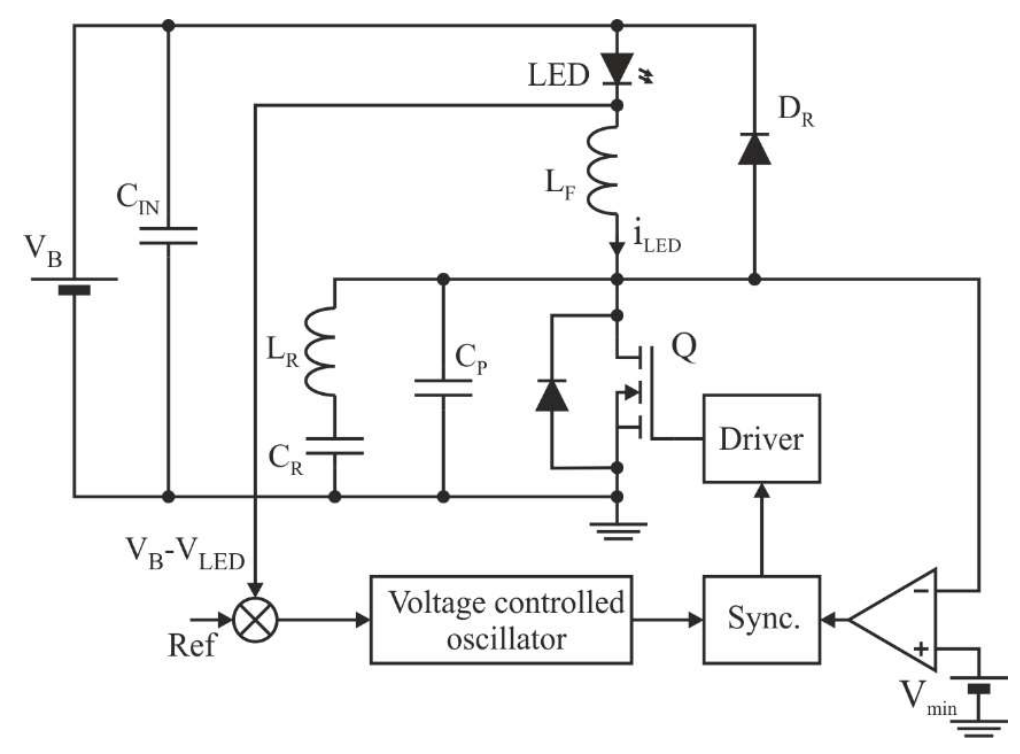

Figure 8. Simplified schematics of the control circuit.

The frequency variation required to compensate for a change in the bus voltage (when $V_{L E D}$ is kept constant) can be calculated directly from Figure $7 \mathrm{a}$,c. With the proposed measurement, a change in $V_{L E D}$ cannot be distinguished from a change in $V_{B}$. However, if $\hat{V}_{L E D}$ is also referred to $V_{B U S O}$ instead of $V_{L E D 0}$ (multiplying by $-k$ ), the curves in Figure $7 \mathrm{~b}$ will be comparable to those in Figure $7 \mathrm{a}$. The small-signal power sensitivity charts against changes in $\hat{V}_{B}$ and $\hat{V}_{L E D}$ (both referred to $V_{B U S 0}$ ) are shown in Figure 9.

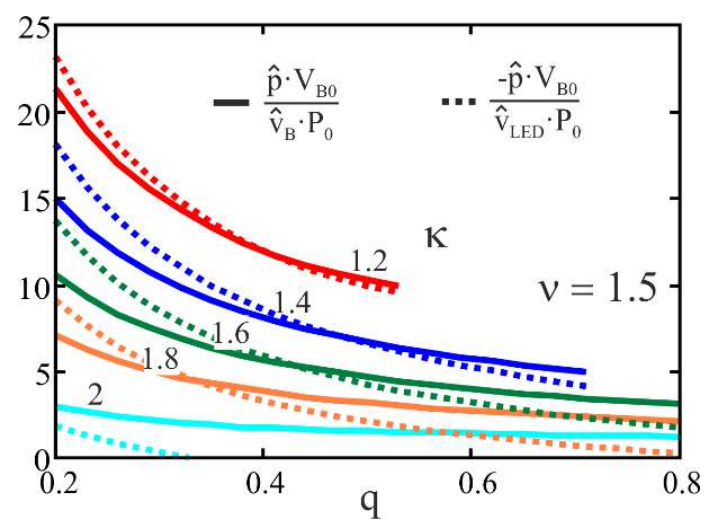

Figure 9. Feedforward control response with $V_{B}$ and $V_{L E D}$ combined.

As it can be observed in the figure, for $q=0.4, k=1.6$ and $v=1.5$, the dashed and solid lines are almost coincident thus the frequency change required to compensate a variation of $1 \mathrm{~V}$ in $V_{B}$ or $-1 \mathrm{~V}$ 
in $V_{L E D}$ will be approximately the same. This characteristic enables sensing $V_{B^{-}} V_{L E D}$ instead of both signals independently. For other values of $q, k$, and $v$, this approximation will be less precise.

Due to the high di/dt values handled at the input of this converter is especially important placing a low ESR (equivalent series resistance) capacitor at the input (shown as $C_{I N}$ in Figure 8).

\section{Design Analysis and Experimental Results}

Using the design example described in Section 4 and the control strategy described previously a laboratory prototype was built and tested. The main characteristics of the circuit components can be found on Table 1.

Transistor switch-on was made by detecting the instant when capacitor $C_{P}$ gets fully discharged. To do so, a threshold value close to zero was compared with $U_{C P}$. This value is shown in Figure 8 as $V_{\min }$. This way, the time interval where the intrinsic mosfet diode is handling current is minimized, further reducing the conduction losses.

Figure 10 shows the main converter waveforms at the nominal operating point. As it can be seen, the ZVS condition is met and capacitor $C_{P}$ is fully discharged when transistor switches on. The LED current has a negligible high frequency ripple due to the high value of the filter inductor $L_{F}$.

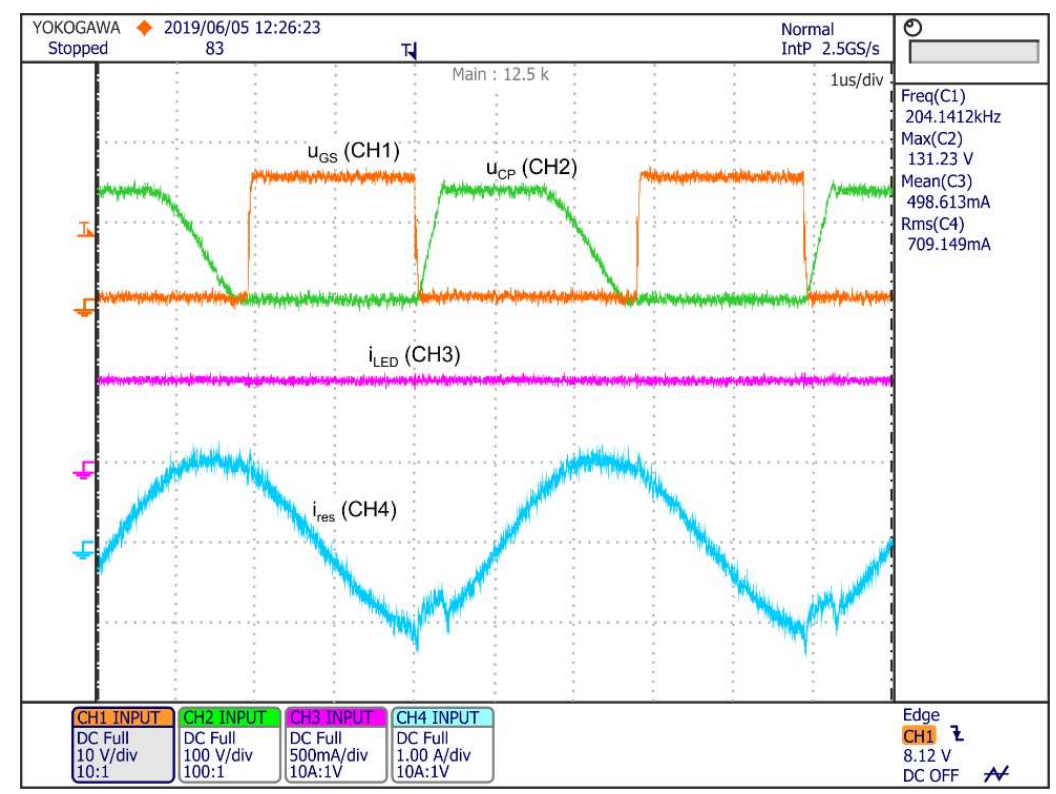

Figure 10. Converter waveforms at nominal operating point.

Figure 11 shows the circuit operating at maximum $V_{B}$ and minimum $V_{L E D}$, which corresponds to $k=2$. It can be observed that the circuit is at the limit of ZVS and $C_{P}$ is not fully discharged when the transistor switches on.

The circuit was also tested at different frequencies for three different values of the bus voltage. The efficiency was measured using a Yokogawa WT3000E Precision Power Analyzer and the results are summarized in Figure 12. As it can be seen, the maximum efficiency is $95.7 \%$ at nominal power. One of the most interesting features observed is that the efficiency is kept above $90 \%$ even when the output power is dimmed below $25 \%$ of its nominal value. The minimum efficiency measured was $77.4 \%$ at $5 \%$ of the rated power. 


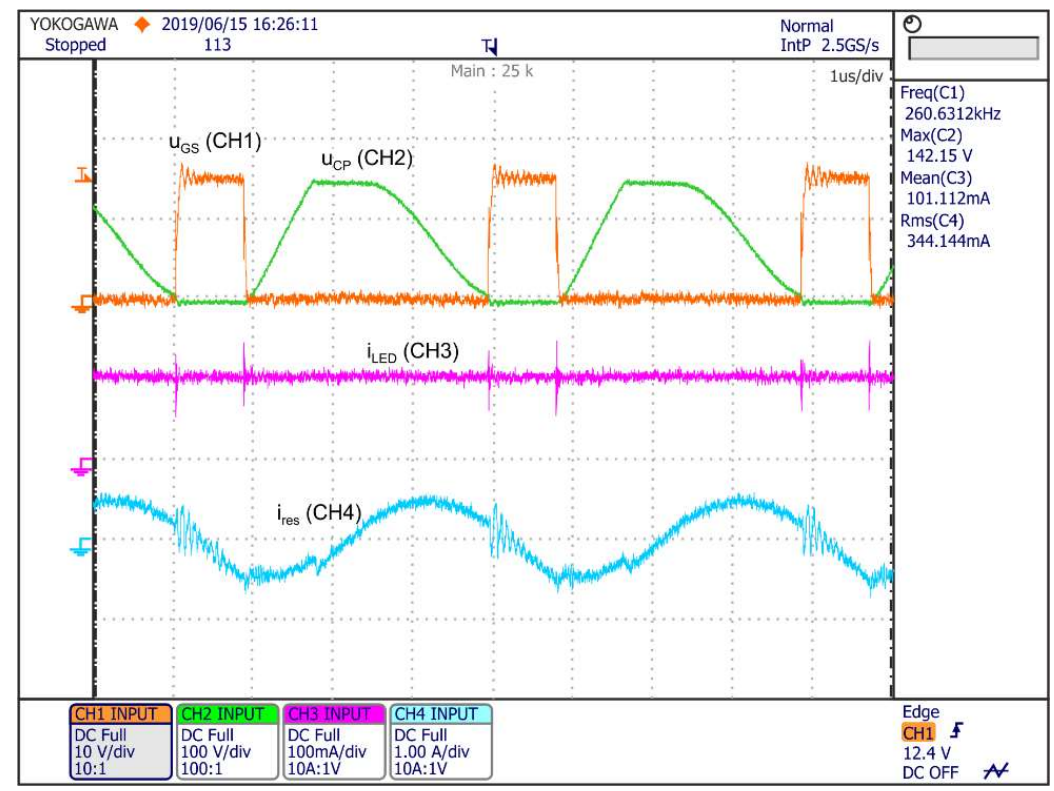

Figure 11. Converter waveforms for $k=2, V_{B}=138 \mathrm{~V}$ and $\mathrm{P}=6.1 \mathrm{~W}$.

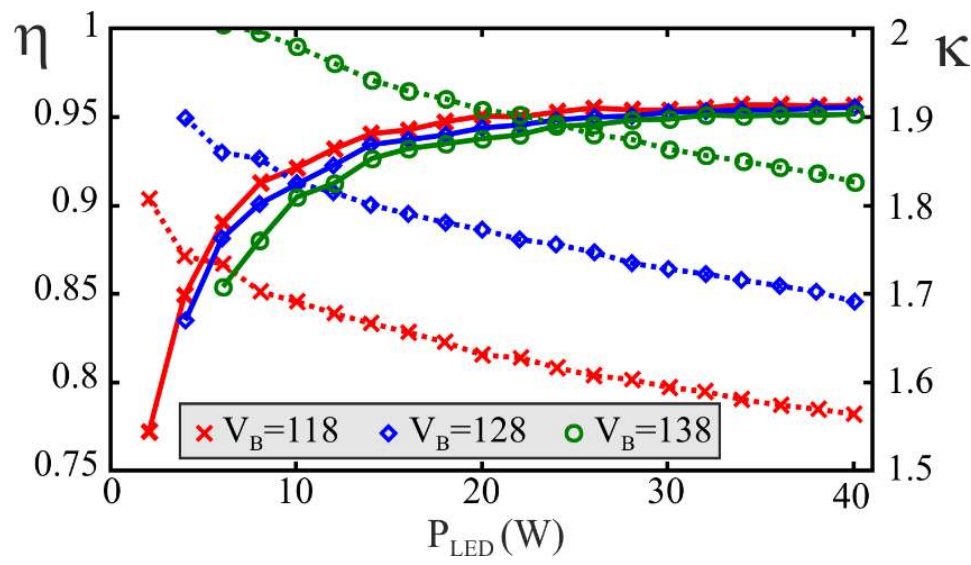

Figure 12. Converter measured efficiency $\eta$ and $k$ value as a function of the output power for three different bus voltages.

Figure 13 shows the lamp power and current at the nominal value of the bus voltage with a $100 \mathrm{~Hz}$ ripple of $16 \mathrm{~V}$ peak. This measurement was made at a constant switching frequency (i.e., with the feedforward control disabled). As it can be seen, there is a very high ripple in the lamp power thus the flicker recommendations of IEEE 1789 are not fulfilled.

Figure 14 shows the same waveforms as the previous figure but with the feedforward control enabled. As it can be observed, the lamp power flicker is highly reduced compared to the previous situation. 


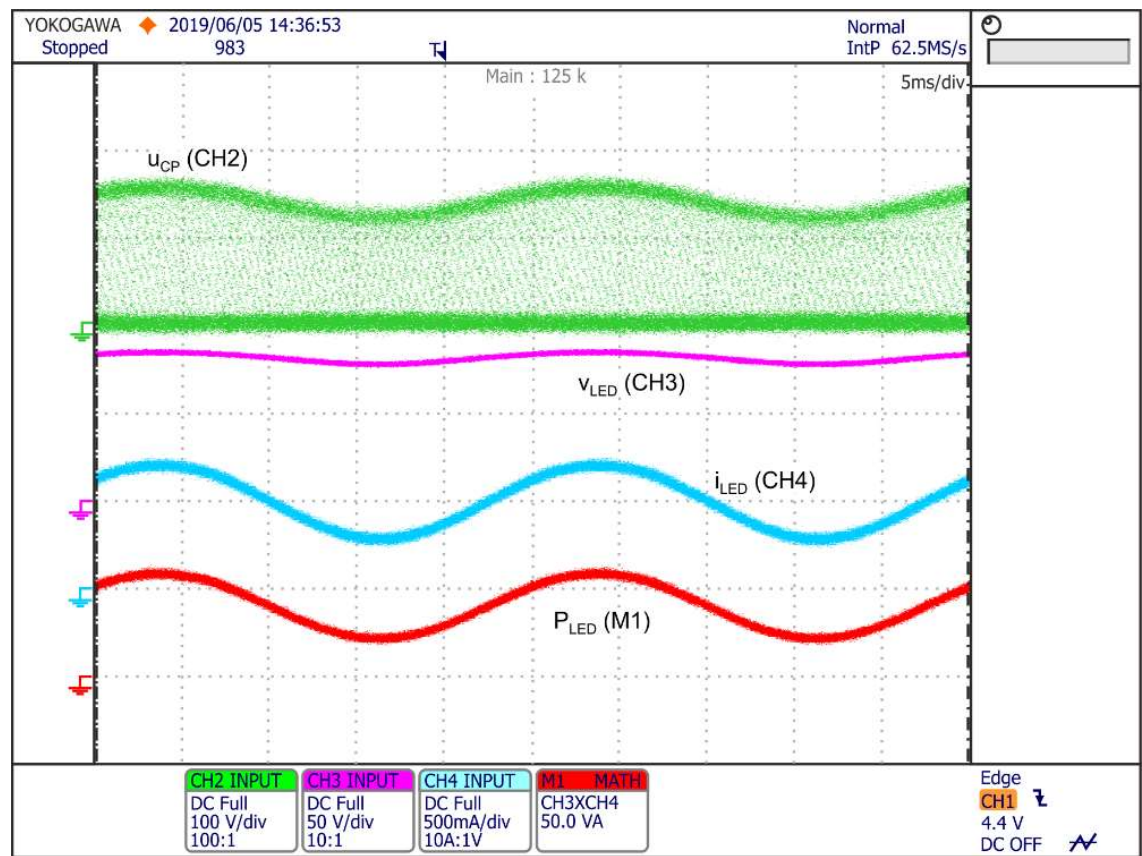

Figure 13. Converter waveforms with a $16 \mathrm{~V}, 100 \mathrm{~Hz}$ ripple in $V_{B}$ with feedforward control disabled.

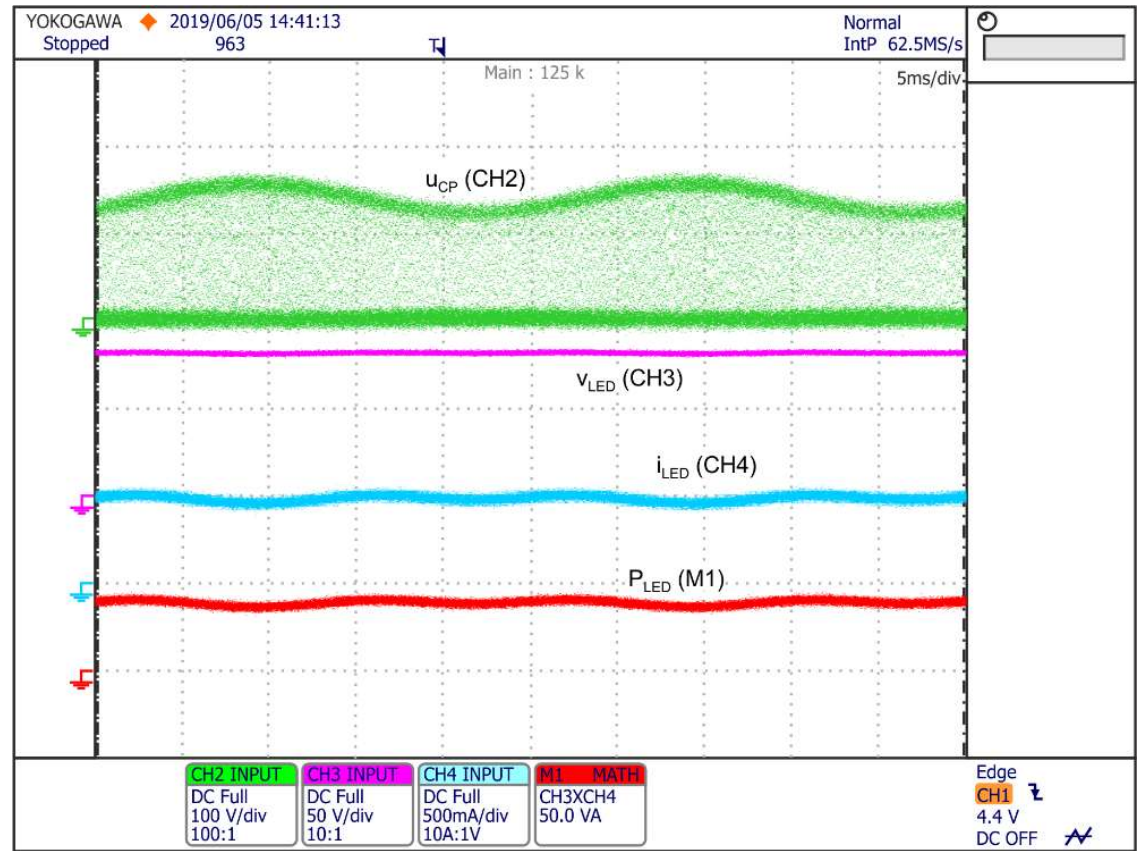

Figure 14. Converter waveforms with a $16 \mathrm{~V}, 100 \mathrm{~Hz}$ ripple in $V_{B}$ with feedforward control enabled.

\section{Conclusions}

In present work, a new power topology based on a Class-E resonant inverter has been presented. The proposed topology works as a LED series current regulator and requires a single controlled switch referred to ground. As in the standard Class-E inverter, this converter is especially suited to operate at very high frequencies due to its extremely small switching losses.

The circuit behavior has been analyzed using the fundamental approach. This analysis has been oriented to obtain a set of charts that allow a straightforward design. Based on the proposed procedure, a design example has been carried out and the results obtained have been used to build a laboratory prototype. 
A simple feedforward control has been used to compensate the variations in the bus and LED voltages. This control changed the switching frequency linearly with the difference between the bus and the lamp voltages. This allowed to compensate variations in both the input and LED voltages using a single voltage sensor. The prototype has been tested with a peak-to-peak input ripple up to $25 \%$ at $100 \mathrm{~Hz}$ without losing ZVS commutations.

The maximum efficiency measured was $95.7 \%$ at nominal power. One of the most interesting features observed during the experimentation is that the efficiency was kept above $90 \%$ even when the output power was dimmed below $25 \%$ of its nominal value.

Author Contributions: Conceptualization, J.R.; writing-original draft preparation, J.R.; methodology, P.J.Q.; writing-review and editing, J.C. and P.J.Q.; validation, A.J.C.; supervision, E.L.-C.; funding acquisition, E.L.-C. and A.J.C.

Funding: This research received no external funding.

Conflicts of Interest: The authors declare no conflict of interest.

\section{References}

1. Vu, N.H.; Pham, T.T.; Shin, S. LED Uniform Illumination Using Double Linear Fresnel Lenses for Energy Saving. Energies 2017, 10, 2091. [CrossRef]

2. Lumileds Holding, B.V. Evaluating the Lifetime Behavior of LED Systems. Lumileds 2016; White Paper 20161201. Available online: https://www.lumileds.com/uploads/167/WP15-pdf (accessed on 13 June 2019).

3. Alexeev, A.; Onushkin, G.; Linnartz, J.P.; Martin, G. Multiple Heat Source Thermal Modeling and Transient Analysis of LEDs. Energies 2019, 12, 1860. [CrossRef]

4. Mathews, E.; Guclu, S.S.; Liu, Q.; Ozcelebi, T.; Lukkien, J.J. The Internet of Lights: An Open Reference Architecture and Implementation for Intelligent Solid State Lighting Systems. Energies 2017, 10, 1187. [CrossRef]

5. Huang, Y.S.; Luo, W.C.; Wang, H.C.; Feng, S.W.; Kuo, C.T.; Lu, C.M. How Smart LEDs Lighting Benefit Color Temperature and Luminosity Transformation. Energies 2017, 10, 518. [CrossRef]

6. Kim, I.T.; Kim, Y.S.; Cho, M.; Nam, H.; Choi, A.; Hwang, T. High-Performance Accuracy of DaylightResponsive Dimming Systems with Illuminance by Distant Luminaires for Energy-Saving Buildings. Energies 2019, 12, 1860. [CrossRef]

7. International Energy Agency IEA. Solid State Lighting Annex: Product Quality and Performance Tiers. Available online: https://ssl.iea-4e.org (accessed on 12 June 2019).

8. Jägerbrand, A. LED (Light-Emitting Diode) Road Lighting in Practice: An Evaluation of Compliance with Regulations and Improvements for Further Energy Savings. Energies 2016, 9, 367. [CrossRef]

9. ANSI/IES TM-30-18. IES Method for Evaluating Light Source Color Rendition; Illuminating Engineering Society: New York, NY, USA, 2018.

10. IEEE Standards Association. IEEE PAR1789: Recommended Practices for Modulating Current in High Brightness LEDs for Mitigating Health Risks to Viewers; IEEE Standards: Piscataway, NJ, USA, 2015.

11. Zhang, F.; Xu, J. A Novel PCCM Boost PFC Converter With Fast Dynamic Response. IEEE Trans. Ind. Electron. 2011, 58, 4207-4216. [CrossRef]

12. Chen, Z.; Zhang, C.; Wu, Y.; Chen, Y.; Zhang, C. Series-Capacitor-Based Buck PFC Converter With High Power Factor and Ultrahigh Step-Down Conversion Ratio. IEEE Trans. Ind. Electron. 2019, 66, 6895-6905. [CrossRef]

13. Niaz, M.T.; Imdad, F.; Kim, H.S. Power Consumption Efficiency Evaluation of Multi-User Full-Duplex Visible Light Communication Systems for Smart Home Technologies. Energies 2017, 10, 254. [CrossRef]

14. Almeida, P.S.; Jorge, J.M.; Rodrigues, C.R.B.S.; Soares, G.M.; Pinto, D.P.; Braga, H.A.C. A Novel Method of Current Equalization in LED Strings Based on Simple Linear Circuit. In Proceedings of the 2011 IEEE International Symposium on Industrial Electronics, Gdansk, Poland, 27-30 June 2011.

15. Han, J.H.; Lim, Y.C. Design of an LLC Resonant Converter for Driving Multiple LED Lights Using Current Balancing of Capacitor and Transformer. Energies 2015, 8, 2125-2144. [CrossRef]

16. Ribas, J.; Quintana-Barcia, P.J.; Cardesin, J.; Calleja, A.J.; Lopez-Corominas, E. LED Series Current Regulator Based on a Modified Class-E Resonant Inverter. IEEE Trans. Ind. Electron. 2018, 65, 9488-9497. [CrossRef] 
17. Dong, H.; Xie, X.; Jiang, L.; Jin, Z.; Zhao, X. An Electrolytic Capacitor-Less High Power Factor LED Driver Based on a "One-and-a-Half Stage" Forward-Flyback Topology. IEEE Trans. Power Electron. 2018, 33, 1572-1584. [CrossRef]

18. Zhang, J.; Jiang, T.; Wu, X. A High-efficiency Quasi-two-stage LED Driver with Multi-channel Outputs. IEEE Trans. Ind. Electron. 2017, 64, 5875-5882. [CrossRef]

19. Almeida, P.S.; Camponogara, D.; Dalla-Costa, M.; Braga, H.; Alonso, J.M. Matching LED and Driver Life Spans: A Review of Different Techniques. IEEE Ind. Electron. Mag. 2015, 9, 36-47. [CrossRef]

20. Nassary, M.; Orabi, M.; Arias, M.; Ahmed, E.M.; Hasaneen, E.S. Analysis and Control of Electrolytic Capacitor-Less LED Driver Based on Harmonic Injection Technique. Energies 2018, 11, 3030. [CrossRef]

21. Liu, K.H.; Lee, F.C.Y. Zero-Voltage Switching Technique in DC/DC Converters. IEEE Trans. Power Electron. 1990, 5, 293-304. [CrossRef]

22. Zhang, W.P.; Zhang, X.Q.; Xiao, S.S. A Novel Soft Switch for Buck Converter. In Proceedings of the IEEE 2nd. International Symposium on Power Electronics for Distributed Generation Systems 2010, Hefei, China, 16-18 June 2010.

23. Bernal, C.; Oyarbide, E.; Molina, P.; Mediano, A. Dynamic Model of Class-E Inverter with Multifrequency Averaged Analysis. IEEE Trans. Ind. Electron. 2012, 59, 3737-3744. [CrossRef]

24. Suetsugu, T.; Kazimierczuk, M.K. Design Procedure for Lossless Voltage-Clamped Class E Amplifier with a Transformer and a Diode. IEEE Trans. Power Electron. 2005, 20, 56-64. [CrossRef]

(C) 2019 by the authors. Licensee MDPI, Basel, Switzerland. This article is an open access article distributed under the terms and conditions of the Creative Commons Attribution (CC BY) license (http://creativecommons.org/licenses/by/4.0/). 